\title{
3D steering of a flexible needle by visual servoing
}

\author{
Alexandre Krupa \\ Inria Rennes - Bretagne Atlantique and IRISA, F-35042 Rennes Cedex, France \\ Alexandre.Krupa@inria.fr \\ http://www.irisa.fr/lagadic/welcome-eng.html
}

\begin{abstract}
This paper presents a robotic control method for 3D steering of a beveled-tip flexible needle. The solution is based on a new dutycycling control strategy that makes possible to control three degrees of freedom of the needle. A visual servoing control scheme using two orthogonal cameras observing a translucent phantom is then proposed to automatically steer a needle toward a 3D target point. Experimental results show a final positioning error of $0.4 \mathrm{~mm}$ and demonstrate the feasibility of this promising approach and its robustness to model errors.
\end{abstract}

Keywords: Flexible needle steering, visual servoing

\section{Introduction}

The needle is a tool used in many medical procedures as for example aspiration biopsy for cancer diagnosis. However, steering a needle requires a lot of skills from the clinician in order to reach the target successfully. A new community of researchers are currently working to provide robotic assistance solutions and more particularly for steering bevel-tip flexible needles [1]. By applying a translation motion to this kind of needle, this later bends during the insertion due to asymmetric forces exerted by the soft tissue onto the beveled tip. As a consequence the needle follows an arc in a 2D plane as reported in $[2,3]$. This bending becomes an advantage for targeting anatomical elements that were not possible to reach with a standard rigid needle. Several research works have therefore been conducted to automatically steer a flexible needle actuated by a robot. In most approaches a path planning initial step based on inverse kinematics is required to predict the needle trajectory. A finite element mesh was also considered in [4] to predict soft tissue deformations and plan the path thanks to a numerical optimization. Probabilistic methods were also tested, like the RapidlyExploring-Random Tree (RRT) algorithm [5], or the Stochastic roadmap [6]. In [7] a kinematic model was proposed for bevel-tip needle steering by constantly spinning the needle during its insertion. This model allows proportional control of the needle curvature and was used in a control-loop that online adapts the evolution of the needle trajectory along an arc in a 2D plane [8]. Other approaches based on the RRT algorithm were proposed to take into account obstacles during the path planning. For example, a RRT algorithm with backchaining was presented in [9] to steer the needle in 3D with obstacle avoidance. More recently, 
a combination of the RRT algorithm and duty-cycling control technique was proposed in [10] for needle steering in a $2 \mathrm{D}$ plane. In this paper, we propose a new approach to steer a needle in 3D that has several advantages. First, it does not require a trajectory planning that can be time consuming. Second, it does not constraint the needle to follow a succession of planar arcs but allows non planar 3D trajectories. Third, with the proposed method the direct control of the two lateral angular velocities and the insertion velocity of the needle extremity is possible. This last point is crucial since, in opposite to approaches based on path planning, it opens numerous control scheme possibilities as for example visual control using the classical framework of visual servoing [11]. The paper is organized as follows. Section 2 presents the kinematic model of the flexible needle and recalls the principle of the classic 2D duty-cycling control technique. We present in Section 3 our 3D duty-cycling strategy that allows to control the two lateral angular velocities and the insertion velocity of the needle. Section 4 presents the control scheme we propose for automatic soft tissue targeting using visual servoing. Finally, experimental results on the visual targeting task are reported and discussed in section 5 .

\section{Needle kinematic model and duty-cycling technique}

Let $\mathcal{F}_{n}$ and $\mathcal{F}_{w}$ be respectively a Cartesian frame attached to the needle tip and the world frame as shown in Figure 1a. We describe the 3D pose $\mathbf{p}$ (with $\mathbf{p} \in \mathrm{SE}(3))$ of the needle tip with respect to $\mathcal{F}_{w}$ by the homogeneous $4 \times 4$ transformation matrix :

$$
{ }^{w} \mathbf{M}_{n}=\left[\begin{array}{cc}
{ }^{w} \mathbf{R}_{n}{ }^{w} \mathbf{T}_{n} \\
\mathbf{0}_{[1 \times 3]} & 1
\end{array}\right]
$$

where ${ }^{w} \mathbf{R}_{n}$ is a $3 \times 3$ rotation matrix representing the orientation of $\mathcal{F}_{n}$ with

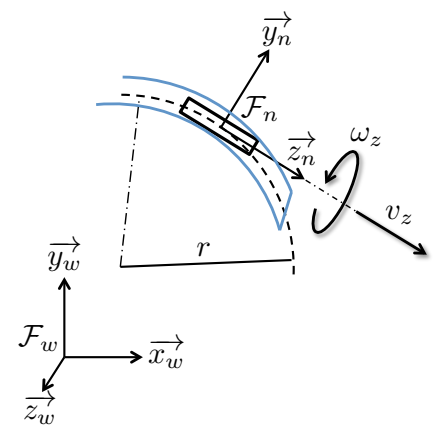

(a)

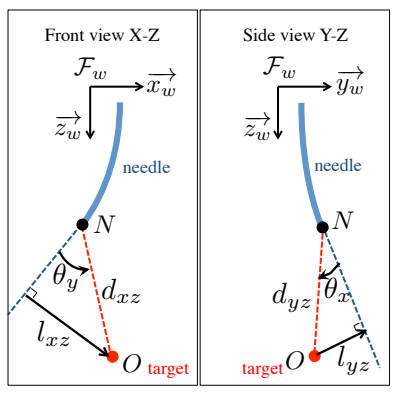

(b)

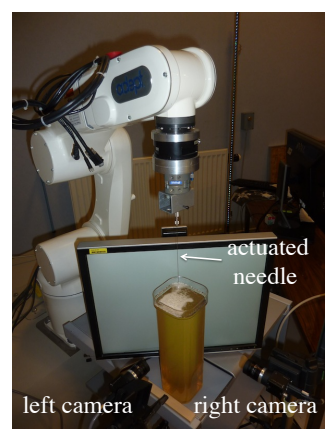

(c)

Fig. 1. (a) needle beveled tip modeled by an unicycle following an arc of radius $r$. (b) Geometry of selected visual features. (c) Experimental setup.

respect to $\mathcal{F}_{w}$ and ${ }^{w} \mathbf{T}_{n}$ is the $3 \times 1$ translation vector defining the origin of $\mathcal{F}_{n}$ in 
$\mathcal{F}_{w}$. We also denote the instantaneous screw velocity vector ${ }^{(n)} \mathbf{v}_{n}$ of the needle frame $\mathcal{F}_{n}$ with respect to the world $\mathcal{F}_{w}$. It is composed by the translational velocities ${ }^{(n)} v_{x},{ }^{(n)} v_{y},{ }^{(n)} v_{z}$ along each axis of $\mathcal{F}_{n}$ and the angular velocities ${ }^{(n)} \omega_{x},{ }^{(n)} \omega_{y},{ }^{(n)} \omega_{z}$ around each axis. Note that the upper script ${ }^{(n)}$ indicates that this velocity is expressed in the frame $\mathcal{F}_{n}$. If we apply a forward insertion motion to the needle, then this later can only move in the plane y-z of $\mathcal{F}_{n}$. Thus, the velocity components ${ }^{(n)} v_{x},{ }^{(n)} v_{y},{ }^{(n)} \omega_{y}$ are constraint to be null and only the insertion velocity ${ }^{(n)} v_{z}$, and angular spin velocity ${ }^{(n)} \omega_{z}$ can be applied by the user to steer the needle. As experimentally demonstrated in [2], the bending effect due to the asymmetric beveled tip introduces a rotation velocity around the $\mathrm{x}$ axis of $\mathcal{F}_{n}$ that depends on the insertion velocity and natural curvature $K$ of the needle such as $\omega_{x}=K^{(n)} v_{z}$. The screw velocity vector is therefore linked to the needle input velocities by the following kinematic relation that expresses the nonholonomic contraints:

$$
{ }^{(n)} \mathbf{v}_{n}=\left[\begin{array}{llllll}
0 & 0 & 1 & K & 0 & 0 \\
0 & 0 & 0 & 0 & 0 & 1
\end{array}\right]^{T}\left(\begin{array}{l}
(n) \\
{ }^{(n)} v_{z} \\
\omega_{z}
\end{array}\right)
$$

The duty cycling method [7] was introduced to allow the proportional control of the curvature of a bevel-tip needle during insertion. It works by alternating between time period $T_{\text {trans }}$ of pure insertion where the needle will follow an arc of maximum curvature $K$, with time period $T_{\text {rot }}$ of both insertion and rotation, where the needle will follow a helical curve. This later becomes a straight line if the rotation velocity is highly superior than the insertion velocity. Those two phases compose a cycle of period $T=T_{\text {rot }}+T_{\text {trans }}$. Therefore, it is possible to obtain a desired effective needle curvature $K_{\text {eff }}=K(1-D C)$ by controlling the duty-cycle ratio $D C=\frac{T_{\text {rot }}}{T}$. In $[8,10]$ this method has been implemented by inserting during each cycle the needle on a constant fixed distance $\Delta z$, and by applying a constant rotational velocity $\omega_{z}=\omega_{c}$ such as:

$$
T_{\text {rot }}=\frac{2 \pi}{\omega_{c}}, T_{\text {trans }}=\frac{T_{\text {rot }}(1-D C)}{D C}, v_{z}=\frac{\Delta z}{T}
$$

However, this technique applies a complete rotation $(2 \pi \mathrm{rad})$ during each cycle of period $T$ which restricts the needle to evolve only in a plane.

\section{3D duty-cycling new strategy}

To overcome this limitation and allow a 3D trajectory that is not composed from a succession of 2D planar arcs, we propose a new duty-cycle strategy. Let us consider a new cartesian frame $\mathcal{F}_{\tilde{n}}$ attached to the needle that corresponds to the current needle frame rotated with an angle $\theta$ around the $z$ axis of $\mathcal{F}_{n}$. By expressing the rotational velocity of $\mathcal{F}_{\tilde{n}}$ in $\mathcal{F}_{n}$ and using the relation (2) with the effective curvature $K_{\text {eff }}$ we obtain the following relation that links the angular velocity of $\mathcal{F}_{n}$ to the needle input velocities ${ }^{(\tilde{n})} v_{z}$ and ${ }^{(\tilde{n})} \omega_{z}$ expressed in $\mathcal{F}_{\tilde{n}}$ :

$$
\left(\begin{array}{l}
{ }^{(n)} \omega_{x} \\
{ }^{(n)} \omega_{y} \\
{ }^{(n)} \omega_{z}
\end{array}\right)=\left[\begin{array}{ccc}
\cos (\theta) & -\sin (\theta) & 0 \\
\sin (\theta) & \cos (\theta) & 0 \\
0 & 0 & 1
\end{array}\right]\left(\begin{array}{c}
K_{\text {eff }}{ }^{(\tilde{n})} v_{z} \\
0 \\
{ }^{(\tilde{n})} \omega_{z}
\end{array}\right)
$$


As $\mathcal{F}_{\tilde{n}}$ and $\mathcal{F}_{n}$ have the same origin and their z-axis are aligned, we have ${ }^{(n)} v_{z}=$ ${ }^{(\tilde{n})} v_{z}$ that we replace in (4) to formulate the angular velocity of $\mathcal{F}_{n}$ by:

$$
{ }^{(n)} \omega_{x}=\cos (\theta) K_{e f f}{ }^{(n)} v_{z} \text { and }{ }^{(n)} \omega_{y}=\sin (\theta) K_{e f f}{ }^{(n)} v_{z}
$$

One can notice that it is therefore possible to control the velocities ${ }^{(n)} \omega_{x}$ and

${ }^{(n)} \omega_{y}$ at each cycle period $T$ by applying an angle $\theta$ around its z-axis before inserting the needle and applying then the desired curvature such as:

$$
\theta=\operatorname{atan}\left({ }^{(n)} \omega_{y} /{ }^{(n)} \omega_{x}\right) \text { and } K_{e f f}=\left(\sqrt{{ }^{(n)} \omega_{x}^{2}+{ }^{(n)} \omega_{y}^{2}}\right) /{ }^{(n)} v_{z}
$$

Note that if ${ }^{(n)} v_{z}$ is null we set $K_{\text {eff }}=0$ since the needle is not moving in this case. In practice, our 3D duty-cycling approach is performed by the following successive steps:

1) measure the current absolute needle spin angle: $\theta_{a}$

2 ) compute the desired absolute spin angle to achieve by accumulating $2 \pi \mathrm{rad}$ and the control angle input $\theta$ given by eq. (6) such as: $\theta_{a}=\theta_{a}+2 \pi+\theta$

3 ) compute the desired period $T_{\text {rot }}$ and desired spin angular velocity profile ${ }^{(n)} \omega_{z}$ needed to achieve $\theta_{a}$ based on a trapezoidal acceleration profile with a nominal needle spin velocity of $\omega_{c}$

4) compute the desired insertion distance $\Delta z={ }^{(n)} v_{z} T_{\text {rot }}$

5) perform the rotation and insertion thanks to the position-based robot lowlevel controller to reach the desired angle $\theta_{a}$ and translation $\Delta_{z}$

6) measure the real period $T_{\text {rot }}$ that was taken by the system

7) compute the desired duty-cycle ratio $D C=1-K_{\text {eff }} / K$ with $K_{\text {eff }}$ given by (6), the desired pure translation period $T_{\text {trans }}=T_{\text {rot }}(1-D C) / D C$ and the desired insertion distance $\Delta z={ }^{(n)} v_{z} T_{\text {trans }}$

8) perform the insertion thanks to the position-based robot low-level controller to reach the desired relative translation $\Delta_{z}$

9) go back to step 1) to start a new cycle.

\section{Automatic targeting by visual servoing}

We consider that a 3D image containing the needle is available thanks for example to the use of a 3D ultrasound probe. It is therefore possible to extract the shape of the needle by performing some image processing. We will not detail any method to extract the needle but the reader can refer for example to the RANSAC-based approach proposed in [12]. By the use of such extraction algorithm it is therefore possible to obtain the current location of the needle tip point that we denoted $N$ of coordinates ${ }^{w} \mathbf{T}_{n}=\left(t_{n x}, t_{n y}, t_{n z}\right)$ and its tip direction orientation ${ }^{w} \mathbf{R}_{n}$ with respect to the world frame $\mathcal{F}_{w}$. As the target coordinates that we denote ${ }^{w} \mathbf{T}_{o}=\left(t_{o x}, t_{o y}, t_{o z}\right)$ are expressed in the world frame $\mathcal{F}_{w}$ and not directly in the needle frame $\mathcal{F}_{n}$, it is more suitable to express the needle lateral angular control velocities in the fixed world frame $\mathcal{F}_{w}$. The lateral angular velocities applied to the needle frame will then be obtained from the 
lateral control velocities ${ }^{(w)} \omega_{x},{ }^{(w)} \omega_{y}$ expressed in $\mathcal{F}_{w}$ thanks to the following relation:

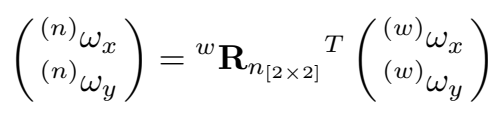

where ${ }^{w} \mathbf{R}_{n_{[2 \times 2]}{ }^{T}}$ is the transpose of the [2 $\left.\times 2\right]$ upper-left part of ${ }^{w} \mathbf{R}_{n}$.

To perform the targeting task we propose to apply a constant needle insertion velocity ${ }^{(n)} v_{z}=v_{c}$ and online adapt the angular control velocities ${ }^{(w)} \omega_{x},{ }^{(w)} \omega_{y}$ by visual servoing in such a way to orientate the needle z-axis toward the target. The set of visual features that we chose is $\mathbf{s}=\left(l_{x z}, l_{y z}\right)$ where $l_{x z}$ and $l_{y z}$ are respectively the distances between the target point $O$ and their orthogonal projections onto the needle line direction projected in the $\mathrm{x}-\mathrm{z}$ plane and $\mathrm{y}-\mathrm{z}$ plane of $\mathcal{F}_{w}$ as shown in Figure 1b. Let us denote $d_{x z}$ and $d_{y z}$ the distances of the projections of vector $\overrightarrow{N O}$ into the $\mathrm{x}-\mathrm{z}$ plane and $\mathrm{y}-\mathrm{z}$ plane of $\mathcal{F}_{w}$. We also define $\theta_{x}$ and $\theta_{y}$ as being respectively the angles around the world frame $\mathrm{x}$ and y axes that describe the relative orientation of the vector $\overrightarrow{N O}$ with respect to needle unitary orientation vector ${ }^{w} \mathbf{u}$ (see Figure $1 \mathrm{~b}$ ). Note that all these geometrical features can be easily computed from the measured 3D positions of the needle and target. The visual features can therefore be obtained from: $l_{x z}=d_{x z} \sin \left(\theta_{y}\right)$ and $l_{y z}=-d_{y z} \sin \left(\theta_{x}\right)$. The visual servoing task consists then in aligning the needle line direction on the target by regulating $\mathbf{s}$ to the desired value $\mathbf{s}^{*}=(0,0)$. As we previously mentioned, one important advantage of our new 3D duty-cycling strategy is that we can apply the classical framework of visual servoing [11]. Therefore we first determine the image jacobian $\mathbf{J}$ that relates the variation $\dot{\mathbf{s}}$ of the selected visual features to the needle velocity inputs and obtain the following expression from basic angular kinematic relationships:

$$
\dot{\mathbf{s}}=\left(\begin{array}{c}
\dot{l_{x z}} \\
\dot{l_{y z}}
\end{array}\right)=\underbrace{\left[\mathbf{J}_{\omega_{x y}} \mathbf{J}_{v_{z}}\right]}_{=\mathbf{J}}\left(\begin{array}{c}
(w) \omega_{x} \\
(w) \omega_{y} \\
(n) v_{z}
\end{array}\right) \text { with } \mathbf{J}_{\omega_{x y}}=\left[\begin{array}{cc}
0 & \cos \left(\theta_{y}\right) d_{x z} \\
-\cos \left(\theta_{x}\right) d_{y z} & 0
\end{array}\right]
$$

and $\mathbf{J}_{v_{z}}=\left[\begin{array}{ll}0 & 0\end{array}\right]^{T}$. Finally we applied the following classical control law that gives the control angular velocity inputs in such a way to obtain an exponential decrease of the visual error $\mathbf{e}=\mathbf{s}-\mathbf{s}^{*}$ :

$$
\left(\begin{array}{l}
{ }^{(w)} \omega_{x} \\
{ }^{(w)} \omega_{y}
\end{array}\right)=-\lambda \mathbf{J}_{\omega_{x y}}^{-1} \mathbf{e} \text { where } \lambda>0 \text { is a proportional gain. }
$$

\section{Experimental results}

The experiment setup is presented in Figure 1c. It is composed of a 6-DOF anthropomorphic robot holding a 22 gauge bevel-tip flexible aspiration biopsy needle of $20 \mathrm{~cm}$ length (Angiotech Chiba MCN2208). A homemade translucent gelatin phantom built relatively less flexible than the needle (assuring the model non-holonomic assumption) is employed to simulate a piece of organic soft tissues and is observed by two orthogonal video cameras. The cameras are used to 


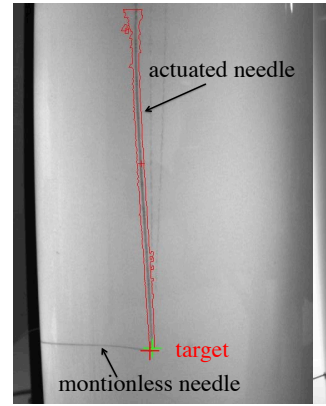

(a)

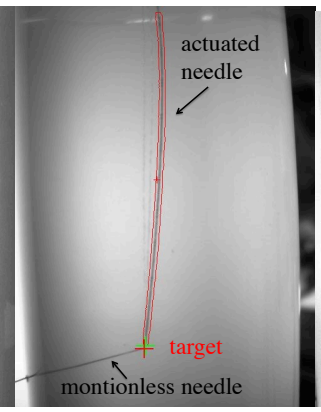

(b)

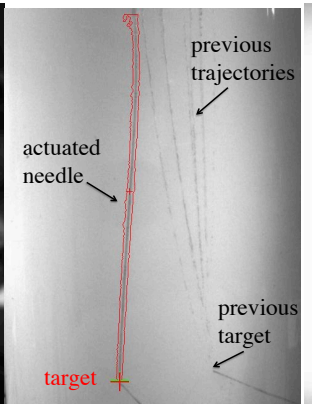

(c)

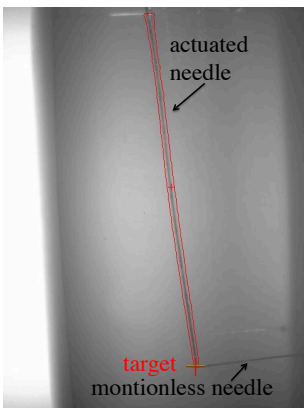

(d)

Fig. 2. (a) right camera ( $x-z$ plane) and (b) left camera (y-z plane) views of the needle trajectory obtained with the targeting task by visual servoing for one trial. (c) right and left $(\mathrm{d})$ views obtained for another trial. The extracted shape of the actuated needle is displayed in red and the needle tip location is shown by the green cross. The red cross corresponds to the target that was defined by indicating in the images the location of the tip of a second motionless needle that was previously manually inserted. One can see the hole in the phantom performed by other previous trials which also successfully reached the target.

perform a 3D localization of the needle tip, but another image modality could be used as for example 3D ultrasound as we plan to consider in future work. The flat screen monitor positioned behind the phantom is used to illuminate the phantom. The image processing and robot motion control is implemented on a PC equiped with a Dual-core 2.4 Ghz Intel Pentium. The needle tip 3D location is measured by first thresholding the images and applying basic morphological operations (erosion, dilatation). Then an algorithm that extracts connected pixels is employed to retain only the pixels corresponding to the needle shape. The tip location and direction vector of the needle is then computed in each image and their 3D coordinates are obtained thanks to the intrinsic parameters of the two calibrated cameras. We coarsely fixed the natural curvature value of the needle that is used in the control to $K=2 \mathrm{~m}^{-1}$ (corresponding to a radius of $0.5 \mathrm{~m}$ ). In all experiments we applied a constant insertion velocity of $1 \mathrm{~mm} / \mathrm{s}$ and the control gain of the visual servoing was empirically set to $\lambda=0.02$. In order to simulate a target point in the phantom we manually inserted another needle from an entry point realized on a lateral wall of the phantom as shown in the bottom of the camera views presented in Figure 2. This allows us to obtain the target 3D coordinates ground truth by clicking on the tip of this motionless needle in the two images. We performed 25 trials of our automatic targeting task by visual servoing with different target positions that were fixed in a $3 \mathrm{D}$ region that is reachable by the needle. For each experiment the entry point of the needle actuated by the robot was slightly shifted for avoiding insertion in a previous hole of the phantom. For all these trials the target was successfully reached. Figure 2 presents the needle trajectories observed in the left and right images 


\begin{tabular}{|c|c|c|c|}
\hline Mean $(\mathrm{mm})$ & Standard deviation $(\mathrm{mm})$ & Max $(\mathrm{mm})$ & Min $(\mathrm{mm})$ \\
\hline 0.432 & 0.262 & 1.023 & 0.113 \\
\hline
\end{tabular}

Table 1. Mean, standard deviation, maximum and minimum values of the final positioning error $d_{\text {error }}$ of the needle tip with respect to the target obtained from the 25 automatic targeting trials.

for two trials. One can see that the target depicted by the red cross is perfectly reached by the needle tip. Table 1 reports the mean, the standard deviation, the maximum and minimum of the final positioning error that are obtained from the 25 trials. This error is defined as the euclidiean norm $d_{\text {error }}$ of the distance between the target and the needle tip. The evolutions of the visual errors for the trial corresponding to the views of Figures 2a-2b are presented in Figures 3a-3b. They correspond as expected to exponential decreases toward zero. Figure $3 \mathrm{c}$ shows the evolution of the distance between the needle tip and target and Figures 3d-3e show the projection of the needle trajectory onto the $\mathrm{x}-\mathrm{z}$ and $\mathrm{y}-\mathrm{z}$ planes of $\mathcal{F}_{w}$. The evolution of the duty-cycling control ratio $D C$ input applied to our 3D duty-cycling low-level controller is reported in Figure 3f. The results also experimentally demonstrate that the targeting task by visual servoing is robust to modelling errors since we do not consider the torsional effect of the needle in its kinematic model and we employ a coarse estimated constant value of its natural curvature.

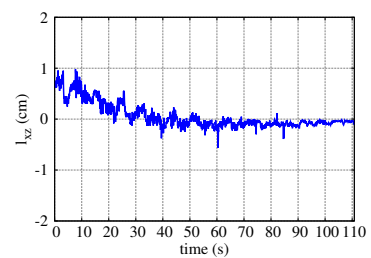

(a) Visual error $l_{x z}$

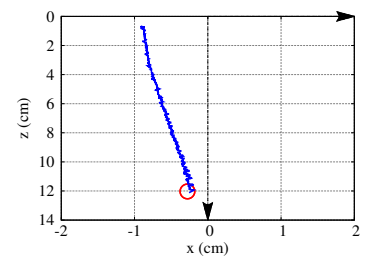

(d) x-z trajectory

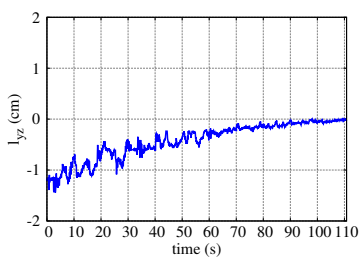

(b) Visual error $l_{y z}$

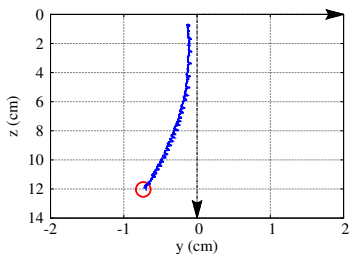

(e) y-z trajectory

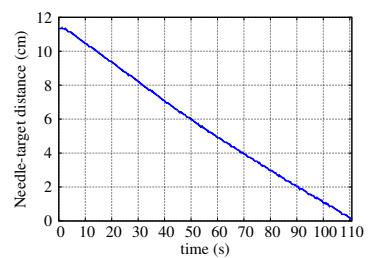

(c) Needle-target distance

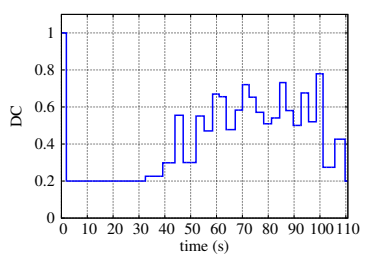

(f) Duty-cycling ratio $D C$

Fig. 3. Results of one trial of the targeting task by visual servoing: (a)-(b) visual features errors. (c) distance between the needle and target. (d)-(e) obtained needle 3D trajectory projected in the $\mathrm{x}-\mathrm{z}$ and $\mathrm{y}-\mathrm{z}$ world frame planes. (f) applied duty-cycling ratio with a lower saturation of 0.2 to limit the period time of pure translation. 


\section{Conclusion}

In this paper we presented a new duty-cycling strategy that allows to control the insertion velocity and the two lateral angular velocities of a bevel-tip flexible needle. Experimental results demonstrate the efficiency of our approach to steer the needle to reach a $3 \mathrm{D}$ target directly by visual servoing without relying like other methods on a computationally expensive path planning that generates a trajectory composed from successive planar arcs. We are currently implementing our approach to automatically steer a flexible needle by ultrasound visual servoing thanks to the use of a 3D ultrasound probe. We are also planning to design a hierarchical multi-task control law for obstacle avoidance.

\section{References}

1. Reed, K., Majewicz, A., Kallem, V., Alterovitz, R., Goldberg, K., Cowan, N., Okamura, A.: Robot-assisted needle steering. IEEE Robotics and Automation Magazine 18(4) (2011) 35-46

2. Webster III, R., Kim, J., Cowan, N., Chirikjian, G., Okamura, A.M.: Nonholonomic modeling of needle steering. The International Journal of Robotics research 25(5-6) (2006) 509-525

3. Misra, S., Reed, K., Schafer, B., Ramesh, K., Okamura, A.: Mechanics of flexible needles robotically steered through soft tissue. The International Journal of Robotics Research 29(13) (2010) 1640-1660

4. Alterovitz, R., Goldberg, K., Okamura, A.: Planning for steerable bevel-tip needle insertion through $2 \mathrm{~d}$ soft tissue with obstacles. In: IEEE Int. Conf on Robotics and Automation. (2005) 1640-1645

5. LaValle, S., Kuffner, J.: Randomized kinodynamic planning. In: IEEE Int. Conf. on Robotics and Automation. Volume 1. (1999) 473-479

6. Alterovitz, R., Simon, T., Goldberg, K.: The stochastic motion roadmap: A sampling framework for planning with markov motion uncertainty. In Robotics: Science and Systems III, W. Burgard and al. (Eds.), MIT Press (2008) 233-241

7. Minhas, D., Engh, J., Fenske, M., Riviere, C.: Modeling of needle steering via duty-cycled spinning. In: IEEE Int. Conf. on Engineering in Medicine and Biology Society. (2007) 2756-2759

8. Wood, N., Shahrour, K., Ost, M., Riviere, C.: Needle steering system using dutycycled rotation for percutaneous kidney access. In: IEEE Int. Conf on Engineering in Medicine and Biology Society (EMBS). (2010) 5432-5435

9. Xu, J., Duindam, V., Alterovitz, R., Goldberg, K.: Motion planning for steerable needles in 3d environments with obstacles using rapidly-exploring random trees and backchaining. In: IEEE Int. Conf on Automation Science and Engineering. (2008) 41-46

10. Bernardes, M., Adorno, B., Poignet, P., Borges, G.: Robot-assisted automatic insertion of steerable needles with closed-loop imaging feedback and intraoperative trajectory replanning. Mechatronics 23 (2013) 630-645

11. Chaumette, F., Hutchinson, S.: Visual servo control, part i: Basic approaches. IEEE Robotics and Automation Magazine 13(4) (2006) 82-90

12. Uhercik, M., Kybic, J., Liebgott, H., Cachard, C.: Model fitting using ransac for surgical tool localization in 3-d ultrasound images. IEEE Trans. on Biomedical Engineering 57(8) (2010) 1907-1916 